\title{
Detection of early adenocarcinoma of the esophagogastric junction by spraying an enzyme-activatable fluorescent probe targeting Dipeptidyl peptidase-IV
}

Keiko Yamamoto', Shunsuke Ohnishi ${ }^{2^{*}}$ (D) Takeshi Mizushima ${ }^{3}$, Junichi Kodaira ${ }^{4}$, Masayoshi Ono ${ }^{5}$, Yutaka Hatanaka ${ }^{6}$, Kanako C. Hatanaka ${ }^{6}$, Yugo Kuriki ${ }^{7}$, Mako Kamiya $^{7}$, Nobuyuki Ehira ${ }^{3}$, Keisuke Shinada $^{8}$, Hiroaki Takahashi ${ }^{4}$,

Yuichi Shimizu', Yasuteru Urano ${ }^{7,9}$ and Naoya Sakamoto ${ }^{2}$

\begin{abstract}
Background: It is still difficult to detect and diagnose early adenocarcinoma of the esophagogastric junction (EGJ) using conventional endoscopy or image-enhanced endoscopy. A glutamylprolyl hydroxymethyl rhodamine green (EP-HMRG) fluorescent probe that can be enzymatically activated to become fluorescent after the cleavage of a dipeptidyl peptidase (DPP)-IV-specific sequence has been developed and is reported to be useful for the detection of squamous cell carcinoma of the head and neck, and esophagus; however, there is a lack of studies that focuses on detecting EGJ adenocarcinoma by fluorescence molecular imaging. Therefore, we investigated the visualization of early EGJ adenocarcinoma by applying EP-HMRG and using clinical samples resected by endoscopic submucosal dissection (ESD).

Methods: Fluorescence imaging with EP-HMRG was performed in 21 clinical samples resected by ESD, and the fluorescence intensity of the tumor and non-tumor regions of interest was prospectively measured. Immunohistochemistry was also performed to determine the expression of DPP-IV.

Results: Fluorescence imaging of the clinical samples showed that the tumor lesions were visualized within a few minutes after the application of EP-HMRG, with a sensitivity, specificity, and accuracy of 85.7, 85.7, and 85.7\%, respectively. However, tumors with a background of intestinal metaplasia did not have a sufficient contrast-tobackground ratio since complete intestinal metaplasia also expresses DPP-IV. Immunohistochemistry measurements revealed that all fluorescent tumor lesions expressed DPP-IV.
\end{abstract}

Conclusions: Fluorescence imaging with EP-HMRG could be useful for the detection of early EGJ adenocarcinoma lesions that do not have a background of intestinal metaplasia.

Keywords: Fluorescence imaging, Dipeptidyl peptidase-IV, Adenocarcinoma of the esophagogastric junction

\footnotetext{
* Correspondence: sonishi@pop.med.hokudai.ac.jp

${ }^{2}$ Department of Gastroenterology and Hepatology, Hokkaido University

Graduate School of Medicine, N15, W7, Kita-ku, Sapporo 060-8638, Japan

Full list of author information is available at the end of the article
}

(C) The Author(s). 2020 Open Access This article is distributed under the terms of the Creative Commons Attribution 4.0 International License (http://creativecommons.org/licenses/by/4.0/), which permits unrestricted use, distribution, and reproduction in any medium, provided you give appropriate credit to the original author(s) and the source, provide a link to the Creative Commons license, and indicate if changes were made. The Creative Commons Public Domain Dedication waiver (http://creativecommons.org/publicdomain/zero/1.0/) applies to the data made available in this article, unless otherwise stated. 


\section{Background}

Adenocarcinoma of the esophagogastric junction (EGJ) is a cancer that develops in the transition zone between the esophagus and the stomach, and its incidence has rapidly increased in recent decades, especially in Western countries [1]. Even in Asia, including Japan, there is concern that the incidence of esophagogastric junctional adenocarcinoma will increase following a decrease in the $H$. pylori infection rate $[2,3]$. Early stage adenocarcinoma of the EGJ can be endoscopically resected, such as by endoscopic submucosal dissection (ESD), and is expected to achieve good oncological outcomes [4-6]. On the other hand, the prognosis of advanced esophageal adenocarcinoma, including esophagogastric adenocarcinoma and Barrett's adenocarcinoma, is poor, and its early detection is necessary for better prognosis [7-9]. Several modalities can detect early carcinoma, such as narrow band imaging (NBI), high magnification chromoendoscopy, indigo carmine dye spraying, and acetic acid spraying [10]. However, detecting early EGJ adenocarcinoma is still difficult because of the following reasons: (1) the tumor develops at a region of physiological construction; (2) the tumor often has a macroscopically flat morphology and exhibits a microsurface structure similar to that of the surrounding non-tumor region; and (3) the tumor sometimes extends under the squamous epithelium [11]. Therefore, the development of a novel and simple method to detect early EGJ adenocarcinoma is needed.

Dipeptidyl peptidase-IV (DPP-IV) is a prolyl-specific protease expressed on the cell surface of a variety of tissues, particularly in the kidney and colon [12]. Certain human cancers, such as those of the prostate, thyroid, and esophagus, show the overexpression of DPP-IV [13-17]. It has recently been reported that glutamylprolyl hydroxymethyl rhodamine green (EP-HMRG), a fluorescent targeting agent based on the fluorophore rhodamine green, becomes fluorescent after cleavage of a DPP-IV-specific sequence, it can be activated within several minutes by topical application in cases of esophageal cancer, and its sensitivity and specificity for diagnosis are comparable to those of iodine chromoendoscopy [18]. In addition, we recently demonstrated that EPHMRG was useful for the rapid detection of superficial head and neck cancer [19]. However, it remains to be elucidated whether EP-HMRG can be applicable as a fluorescent targeting agent in the detection of early EGJ adenocarcinoma. We therefore evaluated whether early EGJ adenocarcinoma could be detected by the application of EP-HMRG with the use of fresh clinical samples obtained by ESD.

\section{Methods}

\section{Definition of carcinoma at the EGJ and Barrett's} esophagus

We defined cancer at the EGJ according to the Japanese classification [20]. In this classification, the area extending $2 \mathrm{~cm}$ above to $2 \mathrm{~cm}$ below the EGJ is designated as the EGJ area. Tumors having their epicenter in this area are designated as EGJ carcinomas irrespective of histological type. The location of an EGJ carcinoma is described using the symbols $E$ (proximal $2 \mathrm{~cm}$ segment) and $\mathrm{G}$ (distal $2 \mathrm{~cm}$ segment), with the dominant area of invasion described first; i.e., $E, E G, E=G$ (both areas equally involved), GE, or G. Barrett's epithelium was endoscopically diagnosed when columnar epithelium was continuously observed from the stomach to the distal side of the esophagus. In the United States of America and most European countries, the diagnosis of Barrett's epithelium requires histologically confirmed intestinal metaplasia [21, 22]. However, in England and Asian countries, including Japan, histological demonstration of goblet cell is not required [23-26]. In this study, we defined Barrett's epithelium as the continuous columnar epithelium from the stomach with or without intestinal metaplasia. The presence of circular Barrett mucosa extending longitudinally for $3 \mathrm{~cm}$ or more in length was classified as long segment Barrett esophagus (LSBE). On the other hand, the presence of circular Barrett mucosa less than $3 \mathrm{~cm}$ in length or the presence of non-circular Barrett mucosa was designated as short segment Barrett esophagus (SSBE) [26].

\section{Enzyme-activatable fluorescent targeting agent}

The EP-HMRG probe was purchased from Goryo Chemical (Sapporo, Japan), resuspended in $10 \mathrm{mM}$ dimethyl sulfoxide (Sigma-Aldrich, St. Louis, MO, USA) and then stored at $80^{\circ} \mathrm{C}$. Before use, the EP-HMRG suspension was thawed to room temperature and diluted to $100 \mu \mathrm{M}$ with phosphatebuffered saline (PBS, Life Technologies, Carlsbad, CA, USA).

\section{Patients}

This study prospectively reviewed early EGJ adenocarcinoma resected by ESD in 23 consecutive patients at five hospitals between May, 2016 and June, 2018. All ESD procedures were performed by experienced endoscopists. We included 21 cases that met the definition of EGJ carcinoma, and excluded the cases in which complete en bloc resection was not performed, as well as cases whose specimens were too damaged for histological investigation.

The resected specimen was immediately extended on a black rubber mat and fixed with pins, and then $100 \mu \mathrm{M}$ EP-HMRG was sprayed onto the specimen. Fluorescence imaging was performed using a handheld fluorescence imaging system (Discovery; INDEC Medical Systems, Santa Clara, CA, USA) that captured white-light images and fluorescence images with $450-490 \mathrm{~nm}$ blue excitation light. The fluorescence images were recorded every minute for $10 \mathrm{~min}$ after the EP-HMRG administration. Subsequently, the specimens were washed with PBS and 
observed using an endoscope (H290Z, Olympus, Tokyo, Japan) under white light.

The fluorescence intensities were measured with ImageJ software (National Institutes of Health, Rockville, MD, USA). We set regions of interest (ROIs) at the area with the most fluorescence signal in the tumor lesion and in the non-tumor region adjacent to the tumor lesion. The mean fluorescence intensity of each ROI was measured as pixel intensity values ranging from 0 to 255 , and the contrast-to-background ratio (CBR) was also measured.

\section{Ethics statement}

The Ethical Review Committee of each hospital approved this ex vivo clinical study protocol. All patients provided informed consent to participate in this study.

\section{Pathological examination}

Specimens were fixed in $40 \mathrm{~g} / \mathrm{L}$ formaldehyde saline, embedded in paraffin, and cut into $5-\mu \mathrm{m}$ sections. Tissue sections were stained with hematoxylin and eosin and then microscopically examined for the histological type, tumor size, depth of invasion, lymphovascular invasion, and resected margin by an experienced pathologist $(\mathrm{KCH})$, according to the World Health Organization classification. Immunohistochemical analysis of DPP-IV expression was performed using an anti-DPP-IV antibody (Novus Biologicals, Littleton, CO, USA). The subtype of intestinal metaplasia in the non-tumor region was determined using the MUC5AC (Agilent, Santa Clara, CA, USA), MUC6 (Abcam, Cambridge, UK), MUC2 (Spring Bioscience, Pleasanton, CA, USA), and CD10 (Agilent) expression patterns. MUC5AC and MUC6 are markers of the gastric phenotype, whereas MUC2 and CD10 are markers of the intestinal phenotype. We defined the complete type as decreased expression of gastric mucin (MUC5AC or MUC6) and coexpression of MUC2 and CD10. The incomplete type was defined as the expression of MUC5AC or MUC6 and MUC2 [27].

Table 1 Patient characteristics

\begin{tabular}{|c|c|c|c|c|c|c|c|c|c|c|c|}
\hline \multirow[t]{2}{*}{ No. } & \multirow{2}{*}{$\begin{array}{l}\text { Age } \\
\text { /Sex }\end{array}$} & \multirow{2}{*}{$\begin{array}{l}\text { Loca } \\
\text {-tion }\end{array}$} & \multicolumn{2}{|c|}{ Non-tumor region adjacent to tumor } & \multirow{2}{*}{$\begin{array}{l}\text { Size } \\
(\mathrm{mm})\end{array}$} & \multirow{2}{*}{$\begin{array}{l}\text { Macroscopic } \\
\text { type }\end{array}$} & \multirow{2}{*}{$\begin{array}{l}\text { Histological } \\
\text { type }\end{array}$} & \multirow[t]{2}{*}{ Depth } & \multirow{2}{*}{$\begin{array}{l}\text { Expression } \\
\text { of DPP-IV } \\
\text { Tumor } \\
\text { /background }\end{array}$} & \multirow{2}{*}{$\begin{array}{l}\text { CBR } \\
\text { at } \\
10 \\
\text { min }\end{array}$} & \multirow{2}{*}{$\begin{array}{l}\text { PT } \\
(\min )\end{array}$} \\
\hline & & & Mucosa & Main type of IM & & & & & & & \\
\hline 1 & $70 \mathrm{~s} / \mathrm{M}$ & $\mathrm{GE}$ & SSBE & - & 23 & $I s+\|a+\| c$ & tub1 > 2 & MM & $+/-$ & 6.4 & 63 \\
\hline 2 & $60 \mathrm{~s} / \mathrm{F}$ & G & Gastric mucosa & complete & 12 & $\| c$ & por2 $>$ tub2 & SM & $+/+$ & 0.5 & 11 \\
\hline 3 & $50 \mathrm{~s} / \mathrm{M}$ & E & SSBE & - & 15 & Ila & tub1 & SMM & $+/-$ & 2.3 & 74 \\
\hline 4 & $60 \mathrm{~s} / \mathrm{M}$ & E & SSBE & - & 10 & $\|c+\| a$ & tub1 & MM & $-1-$ & 1.1 & 33 \\
\hline 5 & $70 \mathrm{~s} / \mathrm{M}$ & G & Gastric mucosa & complete & 28 & $\|a+\| c$ & tub1 & SM & $+/+$ & 1.3 & 80 \\
\hline 6 & $70 \mathrm{~s} / \mathrm{M}$ & E & Squamous epithelium & - & 17 & $11 \mathrm{~b}$ & tub1 & DMM & $+/-$ & 2.1 & 84 \\
\hline 7 & $50 \mathrm{~s} / \mathrm{M}$ & E & LSBE & $\begin{array}{l}\text { income } \\
\text {-plete }\end{array}$ & 7 & $\| c$ & tub1 & MM & $+/+$ & 2.4 & 25 \\
\hline 8 & $80 \mathrm{~s} / \mathrm{M}$ & $E=G$ & SSBE & - & 21 & \|c & tub2 & DMM & $+/-$ & 3.6 & 67 \\
\hline 9 & $60 \mathrm{~s} / \mathrm{F}$ & GE & SSBE & - & 20 & Ila & tub1 & M & $+/-$ & 2.2 & 70 \\
\hline 10 & $70 \mathrm{~s} / \mathrm{F}$ & $E=G$ & Gastric mucosa & - & 23 & Ilc & muc $>$ tub1 & SM & $+/-$ & 2.9 & 27 \\
\hline 11 & $60 \mathrm{~s} / \mathrm{M}$ & EG & Gastric mucosa & complete & 8 & Ila & tub1 > 2 & SMM & $+/+$ & 1.2 & 36 \\
\hline 12 & 80s/M & EG & SSBE & - & 25 & \|c & tub1 & SM & $+/-$ & 6.0 & 41 \\
\hline 13 & $50 \mathrm{~s} / \mathrm{M}$ & GE & Gastric mucosa & - & 9 & \|c & tub1 & M & $+/-$ & 2.2 & 25 \\
\hline 14 & $70 \mathrm{~s} / \mathrm{M}$ & G & Gastric mucosa & - & 10 & I & tub1 > pap & M & $+/-$ & 0.9 & 31 \\
\hline 15 & $60 \mathrm{~s} / \mathrm{M}$ & EG & SSBE & - & 48 & $\|c+\| a$ & pap & M & $+/-$ & 12.6 & 186 \\
\hline 16 & $80 \mathrm{~s} / \mathrm{M}$ & EG & Squamous epithelium & - & 20 & lla & pap & M & $+/-$ & 2.7 & 36 \\
\hline 17 & $40 \mathrm{~s} / \mathrm{M}$ & G & Gastric mucosa & - & 28 & $\|a+\| c$ & tub2 $>1$ & SM & $+/-$ & 2.9 & 53 \\
\hline 18 & $60 \mathrm{~s} / \mathrm{M}$ & EG & Squamous epithelium & - & 19 & IIC & tub1 & DMM & $+/-$ & 2.0 & 41 \\
\hline 19 & 70s/M & GE & Gastric mucosa & - & 10 & Ila & tub1 $>2$ & MM & $+/-$ & 2.9 & 23 \\
\hline 20 & $80 \mathrm{~s} / \mathrm{F}$ & G & Gastric mucosa & - & 12 & Ila & tub1 & M & $+/-$ & 2.2 & 52 \\
\hline 21 & $50 \mathrm{~s} / \mathrm{M}$ & $\mathrm{EG}$ & Squamous epithelium & - & 25 & $\|a+\| c$ & tub1 & DMM & $+/-$ & 4.7 & 40 \\
\hline
\end{tabular}

Tumor location was determined according to the Japanese classification of gastric carcinoma [20]. CBR Contrast-to-background ratio, SSBE Short-segment Barrett's esophagus, LSBE Long-segment Barrett's esophagus, IM Intestinal metaplasia, MM Muscularis mucosae, SMM Superficial muscularis mucosae, DMM Deep muscularis mucosae, M Mucosa, SM Submucosa, tub Tubular adenocarcinoma, pap Papillary adenocarcinoma, por Poorly differentiated adenocarcinoma, PT Procedure time 


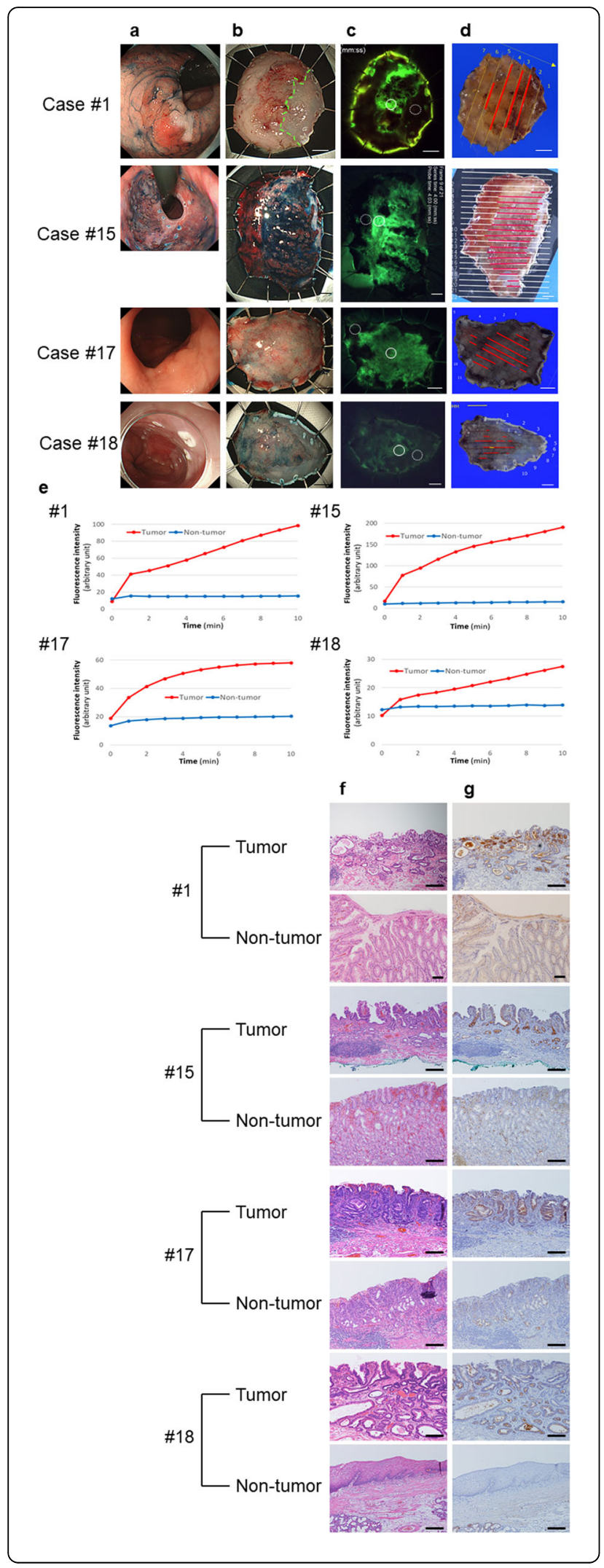

Fig. 1 Fluorescence imaging with EP-HMRG of the four representative cases (cases \#1,\#15, \#17, and \#18). a Endoscopic imaging with white light before endoscopic submucosal dissection (ESD). $\mathbf{b}$ Endoscopic imaging with white light after ESD. The squamocolumnar junction (SCJ) is shown as a green line (case \#1). c Fluorescence imaging after EP-HMRG spraying. The $\mathrm{ROI}$ of the tumor is the solid circle, while the ROl of non-tumor is the dotted circle. $\mathbf{d}$ Resected specimen mapping for the tumor region. The adenocarcinoma is shown as red lines. $\mathbf{e}$ Time course of the fluorescence intensity of the tumor lesion and non-tumor region after spraying EPHMRG. f Hematoxylin and eosin staining and immunohistochemical examination investigating DPP-IV expression in the tumor and non-tumor regions. g Immunohistochemical examination investigating DPP-IV expression in the tumor and non-tumor regions. Scale bars of $\mathbf{b}-\mathbf{d}, 5 \mathrm{~mm}$. Scale bars of $\mathbf{f}$ and $\mathbf{g}, 200 \mu \mathrm{m}$

\section{Statistical analysis}

Receiver operating characteristic (ROC) curves were used to determine the sensitivity, specificity, and accuracy. All analyses were performed using GraphPad Prism version 6 (GraphPad Software, San Diego, CA, USA).

\section{Results}

\section{Patient characteristics}

We included 21 patients with 21 lesions (Table 1). Four lesions were located at the E, six lesions at the EG, two lesions at the $E=G$, four lesions at the $G E$, and five lesions at the $G$, according to the Japanese classification of gastric adenocarcinoma [20]. One lesion developed at long-segment Barrett's esophagus (LSBE, case \#7). In four cases, the cancer lesion was surrounded by complete or incomplete intestinal metaplasia. One lesion developed after radiotherapy (case \#4). Thirteen lesions were $<20 \mathrm{~mm}$ in size, and 19 lesions had differentiated type as the main histological type.

\section{Fluorescence imaging of early EGJ adenocarcinoma}

The resected specimens were sprayed with EP-HMRG, and fluorescence images were obtained every minute for $10 \mathrm{~min}$. The CBRs $10 \mathrm{~min}$ after spraying EP-HMRG are shown in Table 1 . Sixteen of the 21 lesions had sufficient CBRs $(\geq 2)$. Four representative cases (cases \#1, \#15, \#17 and \#18) are shown in Fig. 1. Tumor lesions, but not the non-tumor region, became fluorescent immediately after spraying EPHMRG (Fig. 1c). Histological mapping confirmed that the fluorescent region was almost identical to the cancer lesion (Fig. 1c and d). The fluorescence intensity in the tumor lesions showed a time-dependent increase (Fig. 1e). All representative cases showed sufficient CBRs within a few minutes after EP-HMRG spraying (Table 1). The pathological examination revealed that the tumor lesions expressed DPP-IV, but the non-tumor region did not express DPP-IV (Fig. If and g).

\section{Fluorescence imaging of the tumor extending under the squamous epithelium}

Notably, in several cases (cases \#6, \#8, and \#12), the tumor extended under the squamous epithelium, and most areas of 
the tumor tissue were covered. However conventional endoscopy and NBI showed several point-like structures or small white spots in the squamous epithelium (Fig. 2a and b), and these structures became fluorescent after spraying EPHMRG (Fig. 2d). The pathological examination showed that they were all tumor glands that arised toward the surface and opened in the squamous epithelium (Fig. 2e). The area completely covered by squamous epithelium did not become fluorescent. Columnar epithelial islands without atypia also did not emit fluorescence (Fig. 2, case \#12).

\section{Fluorescence imaging of early EGJ adenocarcinoma with} a background of intestinal metaplasia

In four cases, the tumor lesion developed with a background of intestinal metaplasia. Three of these four cases (cases \#2, \#5, and \#11) had extensive complete intestinal metaplasia in almost all areas adjacent to the tumor lesion. In those cases, both the non-tumor region and the tumor lesion became fluorescent after spraying EP-HMRG (Fig. 3a-e, case \#5). The pathological examination revealed that both the tumor lesion and the intestinal metaplasia expressed DPP-IV (Fig. 3f and g, case \#5). However, the lesion in another case, which arose from LSBE (case \#7), showed a sufficient CBR because the DPP-IV expression in the incomplete intestinal metaplasia was not as strong as a tumor lesion (Fig. 3ag, case \#7).

\section{Diagnostic performance of EP-HMRG for detecting early EGJ carcinoma}

We performed a ROC analysis of the diagnostic performance of EP-HMRG for detecting early EGJ carcinoma. Including all 21 cases, the sensitivity, specificity, and accuracy values were 85.7, 85.7, and 85.7\%,

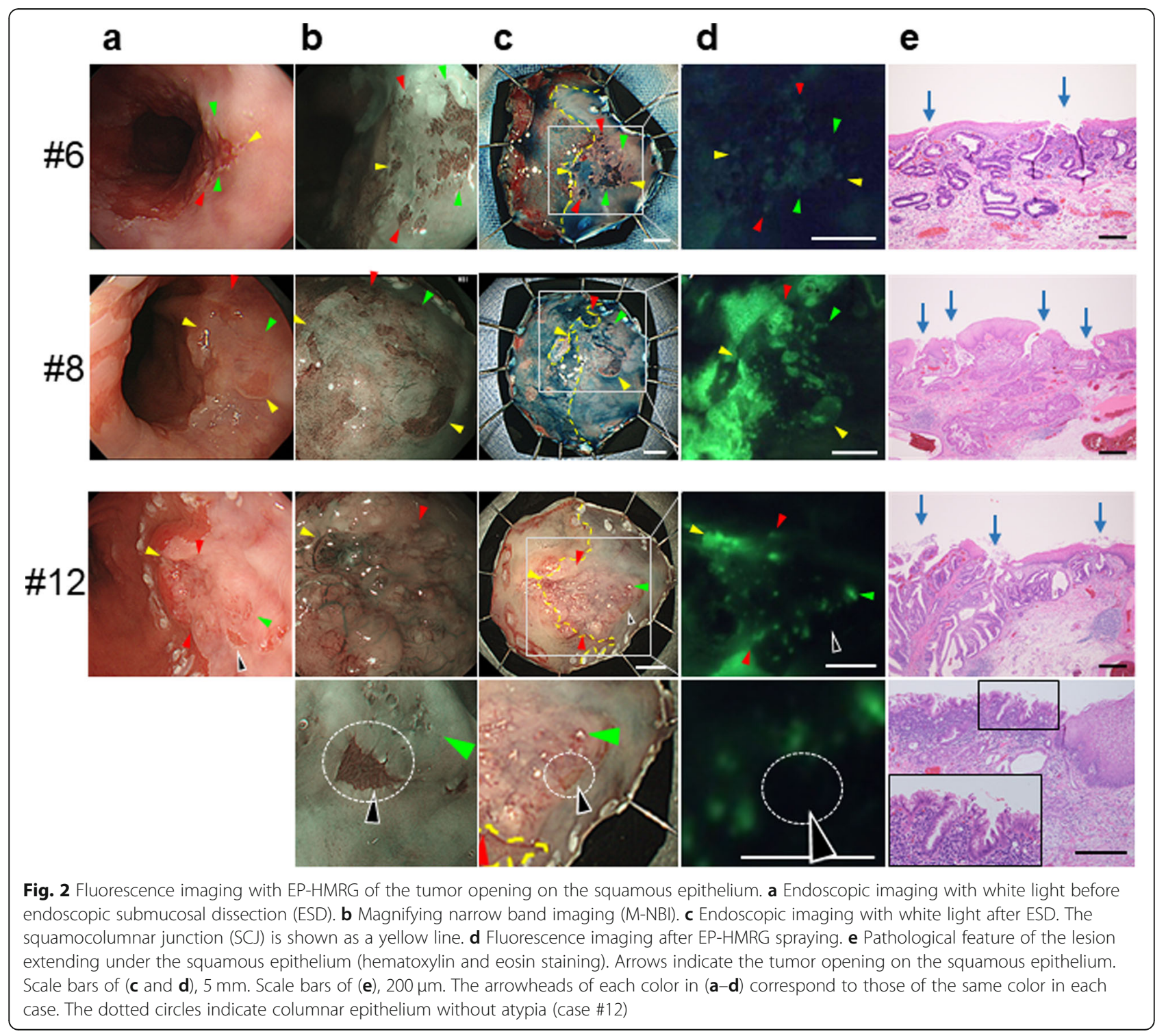




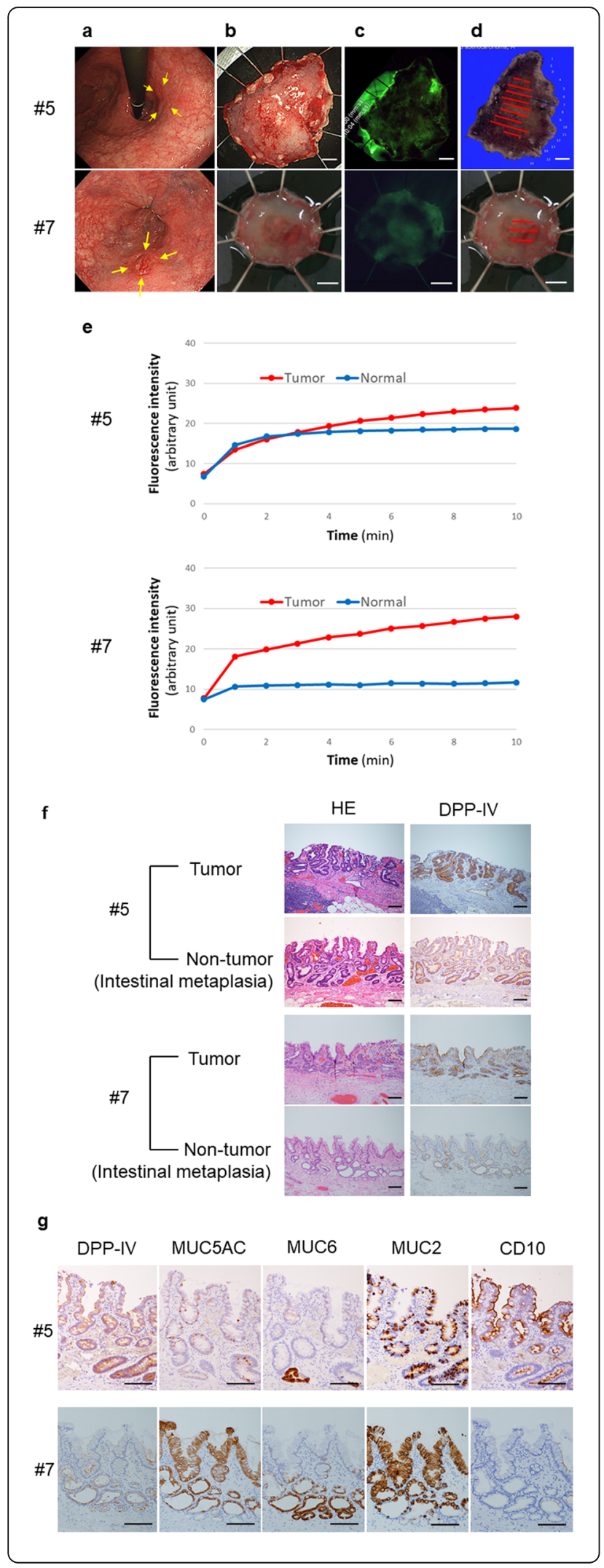

Fig. 3 Fluorescence imaging with EP-HMRG of adenocarcinoma with a background of intestinal metaplasia located at the $\mathrm{G}$ (case \#5) and E with LSBE (case \#7). a Endoscopic imaging with white light before endoscopic submucosal dissection (ESD). b Endoscopic imaging with white light after ESD. c Fluorescence imaging after EPHMRG spraying. d Resected specimen mapping for the tumor region. The adenocarcinoma is shown as red lines. e Time course of the fluorescence intensity of the tumor lesion and the non-tumor region after EP-HMRG spraying. $\mathbf{f}$ Hematoxylin and eosin staining and immunohistochemical examination investigating DPP-IV expression of the tumor lesion and non-tumor region. $\mathbf{g}$ Immunohistochemical examination investigating the subtype of intestinal metaplasia in the non-tumor region. A decrease in the expression of MUC5AC and MUC6 and the positive expression of MUC2 and CD10 indicate complete intestinal metaplasia (\#5). The positive expression of MUC5AC, MUC6, and MUC2 and negative expression of CD10 indicates incomplete intestinal metaplasia (\#7). Scale bars of (b-d), $5 \mathrm{~mm}$. Scale bars of ( $\mathbf{f}$ and $\mathbf{g}$ ), $200 \mu \mathrm{m}$

respectively (area under the curve $[\mathrm{AUC}]=0.85 ; 95 \%$ confidence interval [CI] 0.72-0.98; Fig. 4); however, after excluding the cases with a background of intestinal metaplasia, those values were $88.2,88.2$, and $88.2 \%$, respectively (AUC $=0.86 ; 95 \%$ CI $0.72-1.00$ ). These results demonstrate the usefulness of EP-HMRG in clinical applications, especially for diagnosing lesions without a background of intestinal metaplasia.

\section{Discussion}

In this study, we evaluated the usefulness of EP-HMRG for detecting early EGJ adenocarcinoma and found that the tumor lesion became fluorescent immediately after EP-HMRG spraying and had a sufficient CBR, especially in cases that developed without a background of intestinal metaplasia. To the best of our knowledge, we showed for the first time that EGJ adenocarcinoma can be detected by fluorescence molecular imaging.

Although recently developed modalities, such as NBI and magnifying endoscopy, have been developed for the detection and diagnosis of cancer, extensive endoscopist skills and experience are still necessary for an accurate diagnosis of EGJ adenocarcinoma, including Barrett's adenocarcinoma. In particular, in cases with lesions that extend under the squamous epithelium, it is difficult to detect the lesions. In these cases, glandular spots composed of tumor glands that arise and open on the squamous epithelium are indicative of the existence of carcinoma and give us an opportunity to indicate existence of carcinoma. However, endoscopists often cannot recognize whether these glandular spots are tumor or columnar epithelial islands without atypia, which are found in the lower esophagus within $1 \mathrm{~cm}$ of the squamocolumnar junction in $57 \%$ of patients [28]. This is because most EGJ adenocarcinomas are histologically differentiated, and glandular spots composed of welldifferentiated adenocarcinomas have similar surface 


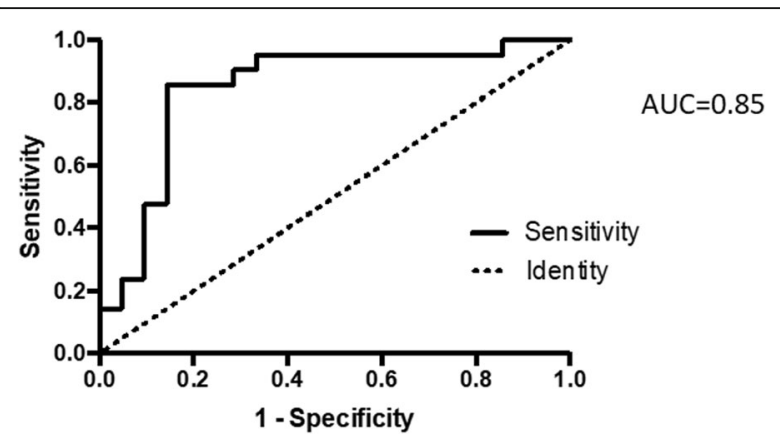

Fig. 4 Receiver operating characteristic (ROC) curves of the EP-HMRG observations of all 21 cases for the detection of early adenocarcinoma of the esophagogastric junction

patterns to columnar epithelial islands. When these spots are very small, endoscopists have difficulty determining whether the glandular spots open on the squamous epithelium are tumor tissue, even with new endoscopic modalities. In the present study, even exceptionally small glandular spots were made more readily visible by EPHMRG. We confirmed that the fluorescence imaging with EP-HMRG enabled the visualization of such cancers that would otherwise be difficult to detect.

It has been reported that DPP-IV is strongly expressed at the brush border of the intestinal metaplasia as well as in gastric cancer [29]. In the present study, all three cases that were entirely surrounded by complete intestinal metaplasia that was characterized by a brush border did not show a sufficient CBR because both the tumor lesion and the intestinal metaplasia region expressed DPP-IV and therefore became fluorescent after EPHMRG spraying. Most gastric cancers in Japan have various degrees of atrophy and intestinal metaplasia following Helicobacter pylori-associated gastritis in the background mucosa. On the other hand, the case of LSBE in the present study (case \#7) with a background of incomplete metaplasia had low expression of DPP-IV; therefore, the CBR was relatively high (2.4). It has been reported that specialized columnar epithelium, which is regarded as a characteristic of Barrett's mucosa in European and American countries, is incomplete intestinal metaplasia without a brush border [30]. Therefore, it is possible that Barrett's adenocarcinoma could be detected by EP-HMRG with a good CBR. Further studies with a larger number of cases will be required to confirm this hypothesis.

Onoyama et al. reported that the EP-HMRG absorption and emission spectra depend on $\mathrm{pH}$, while EPHMRG itself becomes highly fluorescent in acidic conditions [18]. In case \#14, the fluorescence intensity of the background was as high as that of the tumor lesion despite the lack of DPP-IV expression in the background (data not shown). This is the only case in which a pathological feature did not correspond to the CBR, and it appeared that EP-HMRG became fluorescent due to the presence of gastric acid.

In the present study, one patient developed a tumor after radiotherapy (case \#4, Additional file 1: Figure S1); the tumor did not become fluorescent after spraying, and it did not express DPP-IV. Although the reason for this is not clear, we have previously reported in a study of head and neck squamous cell carcinoma that five tumors that developed after radiotherapy did not express DPP-IV and did not show sufficient CBR [19].

There are several limitations to this study. As our study was performed ex vivo, the DPP-IV activity could have potentially decreased following tumor resection. It took $10-20 \mathrm{~min}$ for the fluorescence imaging to initiate after tumor resection, and the imaging was performed at room temperature rather than at $37^{\circ} \mathrm{C}$, which is the condition we previously reported [19]. Therefore, we would expect that the EP-HMRG application would be more active in an in vivo clinical study than in an ex vivo study using resected specimens. Additionally, in cases with morphological 0-IIa or 0-I, fluorescence was not observed in the middle of the raised areas but was observed at the margin of these areas. There is a possibility that the probe flew out to the surroundings due to gravity before incorporated into the cancer cells. Therefore, it appears that an EP-HMRG suspension with high viscosity would resolve these concerns.

However, it is generally easy to detect elevated lesions with conventional endoscopy. Furthermore, most of the cases with elevated portions in this study coexisted with a 0-IIb or 0-IIc component, and fluorescence was observed in these areas.

\section{Conclusions}

In conclusion, our data suggest that the topical spraying of EP-HMRG enabled rapid fluorescence imaging of early EGJ adenocarcinoma. Further studies to validate for cases with intestinal metaplasia and to evaluate the safety of this probe, as well as the development of fluorescence endoscopy to capture the fluorescence emitted by EP-HMRG, are necessary. We expect that many endoscopists will be able to more easily detect EGJ carcinoma at an early stage by applying EP-HMRG.

\section{Supplementary information}

Supplementary information accompanies this paper at https://doi.org/10. 1186/s12885-020-6537-9.

Additional file 1: Supplementary Figure. Fluorescence imaging with EP-HMRG and pathological examination of adenocarcinoma after radiotherapy (case \#4). (a) Endoscopic imaging with white light before endoscopic submucosal dissection (ESD). Arrows indicate the tumor lesion. (b) Endoscopic imaging with white light after ESD. (c) Fluorescence imaging 
after EP-HMRG spraying. (d) Resected specimen mapping for the tumor region. The adenocarcinoma is shown as red lines. (e) Time course of the fluorescence intensity of the tumor lesion and the non-tumor region after EP-HMRG spraying. (f) Hematoxylin and eosin staining of the tumor lesion and the non-tumor region. (g) Immunohistochemical examination investigating DPP-IV expression in the tumor lesion and the non-tumor region. Scale bars of $\mathbf{b}-\mathbf{d}, 5 \mathrm{~mm}$. Scale bars of $\mathbf{f}$ and $\mathbf{g}, 200 \mu \mathrm{m}$.

\section{Abbreviations}

CBR: Contrast-to-background ratio; DMSO: Dimethylsulfoxide; DPP: Dipeptidylpeptidase; EGJ: Esophagogastric junction; ESD: Endoscopic submucosal dissection; HMRG: Hydroxymethyl rhodamine green; PBS: Phosphate-buffered saline; ROI: Regions of interest

\section{Acknowledgments}

An earlier analysis of this study was previously published as an abstract with the following reference:

Yamamoto K, Ohnishi S, Mizushima T, Kodaira J, Ono M, Hatanaka Y, et al. Detection of early adenocarcinoma of the esophagogastric junction by spraying an enzyme-activatable fluorescent probe targeting dipeptidyl peptidase-IV. Gastrointestinal Endoscopy. 2019;89 (suppl 6):AB624.

\section{Authors' contributions}

$\mathrm{KY}$ and SOh performed the experiments and analyses and drafted the manuscript, $\mathrm{KY}$ and TM performed analysis, $\mathrm{KY}$, TM, JK and MO performed ESD, KY, YH and $\mathrm{KCH}$ performed pathological examinations, MK and YK prepared EP-HMRG, MO, NE, KS and $\mathrm{HT}$ performed connection of the multicentre study, YS, NS and YU supenvised the entire project. All authors have read and approved the final manuscript.

\section{Funding}

This study was funded by a Grant-in-Aid (C) from the Japan Society for the Promotion of Science (JSPS, 17 K09343), providing funding to give chemical agents and editorial assistance with the writing of the manuscript. This funding body had no role in study design, data collection, data analysis or data interpretation.

\section{Availability of data and materials}

The datasets used and/or analyzed during the current study are available from the corresponding author on reasonable request.

\section{Ethics approval and consent to participate}

The ex vivo clinical study protocol was approved by the ethical review board of Hokkaido University Hospital. All patients provided informed consent to participate this study under the 'Ethics, consent and permissions' heading. The written informed consent was obtained.

\section{Consent for publication}

All patients provided written informed consent for publication under the 'Consent to publish' heading.

\section{Competing interests}

The authors declare that they have no competing interests.

\section{Author details}

'Division of Endoscopy, Hokkaido University Hospital, N14, W5, Kita-ku, Sapporo 060-8648, Japan. ${ }^{2}$ Department of Gastroenterology and Hepatology, Hokkaido University Graduate School of Medicine, N15, W7, Kita-ku, Sapporo 060-8638, Japan. ${ }^{3}$ Department of Gastroenterology, Japanese Red Cross Kitami Hospital, N6, E2, Kitami 090-8666, Japan. ${ }^{4}$ Department of Gastroenterology, Keiyukai Daini Hospital, N3-7-1, Hondori, Shiroishi-ku, Sapporo 003-0027, Japan. ${ }^{5}$ Department of Gastroenterology, Hakodate Municipal Hospital, 10-1, Minato-cho 1, Hakodate 041-8680, Japan. ${ }^{6}$ Department of Surgical Pathology, Hokkaido University Hospital, N14, W5, Kita-ku, Sapporo 060-8648, Japan. ${ }^{7}$ Laboratory of Chemical Biology and Molecular Imaging, Graduate School of Medicine, University of Tokyo, 7-3-1 Hongo, Bunkyo-ku, Tokyo 113-0033, Japan. ${ }^{8}$ Department of Gastroenterology, Keiwakai Ebetsu Hospital, Ebetsu, 81-81-6, Yoyogi-cho, Ebetsu 069-0817, Japan. ${ }^{9}$ Japan Agency for Medical Research and Development (AMED)-CREST, 7-1 Ootemachi-1, Chiyoda-ku, Tokyo 100-0004, Japan.
Received: 18 June 2019 Accepted: 13 January 2020

Published online: 28 January 2020

\section{References}

1. Hasegawa S, Yoshikawa T. Adenocarcinoma of the esophagogastric junction: incidence, characteristics, and treatment strategies. Gastric Cancer. 2010;13:63-73.

2. Wu JC. Gastroesophageal reflux disease: an Asian perspective. J Gastroenterol Hepatol. 2008;23:1785-93.

3. Hongo M, Nagasaki Y, Shoji T. Epidemiology of esophageal cancer: orient to occident. Effects of chronology, geography and ethnicity. J Gastroenterol Hepatol. 2009;24:729-35.

4. Park CH, Kim EH, Kim HY, Roh YH, Lee YC. Clinical outcomes of endoscopic submucosal dissection for early stage esophagogastric junction cancer: a systematic review and meta-analysis. Dig Liver Dis. 2015;47:37-44.

5. Nagami Y, Ominami M, Otani K, Tanaka F, Taira K, Yamagami H, et al. Endoscopic submucosal dissection for adenocarcinomas of the esophagogastric junction. Digestion. 2018;97:38-44.

6. Gong EJ, Kim DH, Ahn JY, Jung KW, Lee JH, Choi KD, et al. Comparison of long-term outcomes of endoscopic submucosal dissection and surgery for esophagogastric junction adenocarcinoma. Gastric Cancer. 2017;20(Suppl 1): 84-91.

7. Gillison EW, Powel J, McConkey CC, Spychal RT. Surgical workload and outcome after resection for carcinoma of the oesophagus and carcia. Br J Surg. 2002;89:344-8.

8. Inadomi JM, Sampliner R, Lagergre J, Lieberman D, Fendrick M, Vakil N. Screening and surveillance for Barrett esophagus in high-risk groups: a costutility analysis. Ann Intern Med. 2003;138:176-86.

9. Sharman P, Sidorenko El. Are screening and surveillance for Barrett's oesophagus really worthwhile? Gut. 2005;54:27-3.

10. Goda K, Singh R, Oda I, Omae M, Takahashi A, Koike T, et al. Current status of endoscopic diagnosis and treatmentof superficial Barrett's adenocarcinoma in Asia-Pacific region. Dig Endosc. 2013;25(Suppl 2):146-50.

11. Omae M, Fujisaki J, Shimizu T, Igarashi M, Yamamoto N. Magnifying endoscopy with narrow-band imaging finding in the diagnosis of Barrett's esophageal adenocarcinoma spreading below squamous epithelium. Dig Endosc. 2013;25(Suppl 2):162-7.

12. Juillerat-Jeanneret L. Dipeptidyl peptidase IV and its inhibitors: therapeutics for type 2 diabetes and what else? J Med Chem. 2014;57:2197-212.

13. Wilson MJ, Ruhland AR, Quast BJ, Reddy PK, Ewing SL, Sinha AA, et al. Dipeptidylpeptidase IV activities are elevated in prostate cancers and adjacent benign hyperplastic glands. J Androl. 2000;21:220-6.

14. Tanaka T, Umeki K, Yamamoto I, Sakamoto F, Nobuchi S, Ohtaki N. CD26 (dipeptidyl peptidase IV/DPP IV) as a novel molecular marker for differentiated thyroid carcinoma. Int J Cancer. 1995;64:326-31.

15. Goscinski MA, Suo ZH, Nesland JM, Chen WT, Zakrzewska M, Wang J, et al. Seprase, dipeptidyl peptidase IV and urokinase-type plasminogen activator expression in dysplasia and invasive squamous cell carcinoma of the esophagus. A study of 229 cases from Anyang tumor hospital, Henan Province, China. Oncology. 2008;75:49-59.

16. Augoff K, Hryniewicz-Jankowska A, Tabola R, Czapla L, Szelachowski P, Wierzbicki J, et al. Upregulated expression and activation of membraneassociated proteases in esophageal squamous cell carcinoma. Oncol Rep. 2014;31:2820-6.

17. Pro B, Dang NH. CD26/dipeptidyl peptidase IV and its role in cancer. Histol Histopathol. 2004;19:1345-51.

18. Onoyama H, Kamiya M, Kuriki Y, Komatsu T, Abe H, Tsuji Y, et al. Rapid and sensitive detection of early esophageal squamous cell carcinoma with fluorescence probe targeting dipeptidylpeptidase IV. Sci Rep. 2016;6:26399.

19. Mizushima T, Ohnishi S, Shimizu Y, Hatanaka Y, Hatanaka KC, Kuriki Y, et al. Rapid detection of superficial head and neck squamous cell carcinoma by topically spraying fluorescent probe targeting dipeptidyl peptidase-IV. Head Neck. 2018:40:1466-75.

20. Japanese Gastric Cancer Association. Japanese classification of gastric carcinoma: 3rd English edition. Gastric Cancer. 2011;14:101-12.

21. Shaheen NJ, Falk GW, Iyer PG. Gerson LB; American College of Gastroenterology. ACG clinical guideline: diagnosis and Management of Barrett's esophagus. Am J Gastroenterol. 2016;111:30-50.

22. Spechler SJ, Sharma P, Souza RF, Inadomi JM, Shaheen NJ. American Gastroenterological Association medical position statement on the management of Barrett's esophagus. Gastroenterology. 2011;140:1084-91. 
23. Ogiya K, Kawano T, Ito E, Nakajima Y, Kawada K, Nishikage T, et al. Lower esophageal palisade vessels and the definition of Barrett's esophagus. Dis Esophagus. 2008;21:645-9.

24. Playford RJ. New British Society of Gastroenterology (BSG) guidelines for the diagnosis and management of Barrett's oesophagus. Gut. 2006;55:442.

25. Fitzgerald RC, di Pietro M, Ragunath K, Ragunath K, Ang Y, Kang JY, et al. British Society of Gastroenterology guidelines on the diagnosis and management of Barrett's oesophagus. Gut. 2014;63:7-42.

26. Japan Esophageal Society. Japanese classification of esophageal Cancer, 11th edition: part I. Esophagus. 2017;14:1-36.

27. Nagata N, Shimbo T, Akiyama J, Nakashima R, Kim HH, Yoshida T, et al. Predictability of gastric intestinal metaplasia by mottled patchy erythema seen on endoscopy. Gastroenterology Res. 2011;4:203-9.

28. Nakanishi Y, Saka M, Eguchi T, Sekine S, Taniguchi H, Shimoda T. Distribution and significance of the oesophageal and gastric cardiac mucosae: a study of 131 operation specimens. Histopathology. 2007;51:515-9.

29. Carl-McGrath S, Lendeckel U, Ebert M, Wolter AB, Roessner A, Röcken C. The ectopeptidases CD10, CD13, CD26, and CD143 are upregulated in gastric cancer. Int J Oncol. 2004;25:1223-32.

30. Spechler SJ, Goyal RK. Barrett's esophagus. N Engl J Med. 1986;315:362-71.

\section{Publisher's Note}

Springer Nature remains neutral with regard to jurisdictional claims in published maps and institutional affiliations.

Ready to submit your research? Choose BMC and benefit from:

- fast, convenient online submission

- thorough peer review by experienced researchers in your field

- rapid publication on acceptance

- support for research data, including large and complex data types

- gold Open Access which fosters wider collaboration and increased citations

- maximum visibility for your research: over $100 \mathrm{M}$ website views per year

At BMC, research is always in progress.

Learn more biomedcentral.com/submissions 